\title{
Comparação do processo de soldagem TIG e A-TIG em um aço baixo carbono com microadição de molibdênio
}

\author{
Mônica Aline Magalhães Gurgel ${ }^{1^{*}}$ (D) \\ Ailanderson José Magalhães Gurgel ${ }^{2}$ \\ Vinicius Aleixo Silvestre ${ }^{3}$ \\ Frank de Mello Liberato ${ }^{4}$ \\ Andersan dos Santos Paula ${ }^{5}$
}

\section{Resumo}

A soldagem TIG apresenta algumas limitações como a baixa taxa de deposição e penetração rasa nas juntas soldadas. Para suprir essas limitações foi desenvolvido o processo de soldagem A-TIG (Active Flux TIG Welding) onde aplica-se uma fina camada de fluxo sobre a superfície do material antes de ser soldado. Esse fluxo permite, através de mecanismos como a constrição do arco ou a convecção de Marangoni, aumentar a profundidade do cordão de solda. Desta forma, o objetivo deste trabalho é avaliar o incremento na profundidade, comparando a soldagem TIG e A-TIG com fluxo de $\mathrm{Cr}_{2} \mathrm{O}_{3}$, verificar as características morfológicas da microestrutura e os possíveis efeitos sobre a Ultramicrodureza ao longo da espessura do cordão de solda, em um aço baixo carbono com microadição de molibdênio. Os resultados indicam um ganho em termos de profundidade em comparação com o perfil geométrico da soldagem sem fluxo (TIG), o que corrobora para a boa eficiência do processo de soldagem A-TIG. A microestrutura encontrada na região central da zona fundida do cordão de solda consistiu em ferrita acicular (FA) e ferrita primária (FP) na soldagem TIG, como também a evidência da ferrita com segunda fase (FS) em conjunto com as demais fases na soldagem A-TIG devido à menor taxa de resfriamento resultante de uma maior energia de soldagem neste processo. Pela análise de Ultramicrodureza observou-se os maiores valores de dureza Berkovich (HT-115) e menores de dureza da indentação (Hit), para a soldagem A-TIG, na região do reforço do cordão de solda em relação a raiz, sendo observada influência da profundidade da solda nessas propriedades pelas fases resultantes na microestrutura após resfriamento com menor taxa, devido à maior energia de soldagem.

Palavras-chave: Soldagem; TIG; A-TIG; Fluxo ativo; Ultramicrodureza.

\section{Comparison of the TIG and A-TIG welding process in a low carbon steel with molybdenum microadition}

\begin{abstract}
TIG welding has some limitations, such as the low deposition rate and shallow penetration in the welded joints. To overcome these limitations, the A-TIG (Active Flux TIG Welding) welding process was developed, where a thin flux layer is applied to the material surface before being welded. This flux allows, through mechanisms such as the constriction of the arc or the Marangoni convection, to increase the depth of the weld bead. In this way, the objective of this work is to evaluate the increase in depth, comparing the TIG and A-TIG welding with $\mathrm{Cr}_{2} \mathrm{O}_{3}$ flux, to verify the morphological characteristics of the microstructure and the possible effects on the Ultramicrohardness along the thickness of the weld bead in a low carbon steel with molybdenum microadition. The results indicate a gain in terms of depth compared to the geometric profile of welding without flux (TIG), which corroborates the good efficiency of the A-TIG welding process. The microstructure found in the central region fused weld bead consisted of acicular ferrite (FA) and primary ferrite (FP) in TIG welding, as well as evidence of ferrite with second phase (FS) in conjunction with the other phases in welding A-TIG due to the lower cooling rate resulting from higher welding energy in this process. The Ultramicrohardness analysis showed the highest hardness values of Berkovich hardness (HT-115) and lower indentation hardness (Hit), for A-TIG welding, in
\end{abstract}

\footnotetext{
1,5 Instituto Militar de Engenharia-IME, Rio de Janeiro, RJ, Brasil.

${ }^{2-4}$ Instituto Federal de Minas Gerais - IFMG, Congonhas, MG, Brasil.

*Autor correspondente: monicalinemg@hotmail.com
}

2176-1523 (C) 2021. Gurgel et al. Publicado pela ABM. Este é um artigo publicado em acesso aberto (Open Access) sob a licença Creative Commons Attribution, que permite uso, distribuição e reprodução em qualquer meio, sem restrições desde que o trabalho original seja commons Attribution, 
the region of the weld bead reinforcement in relation to the root, with influence of the weld depth on these property by the phases resulting in the cooled molten microstructure with a lower rate due to higher welding energy.

Keywords: Welding; TIG; A-TIG; Active Flux; Ultramicrohardness.

\section{Introdução}

A soldagem A-TIG (Active Flux TIG Welding) foi desenvolvida pela Paton Electric Welding Institute (PWI) na década de 60 e promove um aumento significativo na penetração do cordão de solda a partir da aplicação de um fluxo de ativação (óxidos e haletos), que é uma mistura de material inorgânico suspenso em meio volátil, na superfície do material antes da soldagem [1,2]. Essa técnica permite suprir algumas limitações do processo TIG (Tungsten Inert Gas), como a baixa produtividade devido à taxa de deposição e penetração rasa. No processo A-TIG tem-se um aumento significativo na produtividade, devido à redução no número de passes de solda, além de não ser necessário a preparação da borda. A capacidade de penetração é de até $300 \%$ em comparação com o processo de soldagem TIG [3]. Segundo Azevedo [4], esse fluxo é formado por uma solução de óxidos ou fluoretos misturados a um solvente, que pode ser acetona, álcool ou mesmo água, a fim de formar uma pasta. A deposição do fluxo é feita por meio de pincel ou spray de forma a se obter uma camada homogênea, e a soldagem é realizada após a secagem [5-7]. Modenesi e colaboradores [8] avaliaram fluxos com composições diferentes procurando justificar a utilização de cada fluxo. Este autor demonstrou que mesmo fluxos simples formados de apenas um componente como $\mathrm{SiO}_{2}$ (dióxido de silício), $\mathrm{TiO}_{2}$ (dióxido de titânio) e $\mathrm{Cr}_{2} \mathrm{O}_{3}$ (óxido de cromo) já são capazes de causar importantes acréscimos de penetração, sem necessidade de misturar-se com outros óxidos [9].

Vários mecanismos foram propostos a fim de justificar a melhoria da profundidade de penetração na soldagem A-TIG. Lucas e Howse [5], após observarem a constrição do arco e o aumento da penetração na poça de solda, deduziram que esta constrição leva a um incremento na densidade de corrente que proporciona um arco mais direcionado agindo na poça de solda o que promove uma maior profundidade de penetração. Esses autores propõem que os elétrons presentes nos vapores ionizados dos ingredientes do fluxo sejam capturados na periferia do arco e, assim, o fluxo da corrente fica restrito ao centro do arco, proporcionando a formação de uma poça de fusão estreita, porém mais profunda. A constrição do arco depende da capacidade do fluxo de combinar-se com os elétrons [5]. Berthier e colaboradores [10] avaliaram os fenômenos de constrição do arco para diferentes fluxos a partir da visualização da forma do arco e observaram que essa constrição é mais importante para um fluxo constituído de apenas um óxido $\left(\mathrm{TiO}_{2}, \mathrm{Cr}_{2} \mathrm{O}_{3}\right.$ ou $\left.\mathrm{K}_{2} \mathrm{Cr}_{2} \mathrm{O}_{7}\right)$ em comparação com o fluxo comercial [10]. Outro mecanismo para justificar o aumento da penetração foi proposto por Heiple e Roper é a convecção de Marangoni [11]. Eles sugerem que o aumento da presença dos elementos ativos provoca uma inversão do gradiente de tensão superficial de negativo para positivo. Assim, a tensão superficial torna-se maior nos pontos de maior temperatura da poça de fusão, ou seja, no centro em relação as bordas, tornando possível a obtenção de uma maior penetração por causa do movimento de convecção (da região de menor tensão superficial para a maior). Entretanto, se o teor de elementos ativos não for suficiente para alterar esse gradiente, a tensão superficial diminui com o aumento da temperatura e o fluxo ocorre do centro para as bordas, produzindo uma poça larga e rasa [12], que é mais adequada quando o objetivo é utilizar a soldagem para serviços de revestimento. Deste modo, a convecção de Marangoni possui direção e magnitude controladas pelo gradiente de tensão superficial com a temperatura [11]. No TIG, os movimentos de Marangoni são centrífugos e não favorecem a penetração do cordão de solda. Já no processo A-TIG, com elementos de tensão superficial ativos (em geral, os elementos da coluna VII da tabela periódica dos elementos químicos), os movimentos de Marangoni tornam-se centrípetos o que potencializa a penetração.

Portanto, o fluxo de ativação pode atuar na física do arco. A dissociação e ionização dos elementos de fluxo promovem os mecanismos que resultam no aumento da densidade de energia do arco e, portanto, na penetração da soldagem. Foi descoberto que os haletos atuam como elementos ativos de superfície e atuam na física do arco. Os fluoretos dissociados possuem grande afinidade com os elétrons, o que promove a constrição do arco $[13,14]$.

Outras pesquisas realizadas $[4,5,8,15]$ também mostraram que os componentes do fluxo vaporizados pelo arco elétrico, no processo de soldagem A-TIG, causariam o mecanismo de constrição. Abinaya e colaboradores [16] discutiram o processo A-TIG e confirmam que o mecanismo de constrição do arco desempenha um papel importante no cordão de solda aumentando a profundidade de penetração ao restringir o arco. O mecanismo de convecção do Marangoni dá baixa largura e maior profundidade devido à mudança na direção da poça de fusão. Deste modo o processo A-TIG possui diversas vantagens, incluindo alta produtividade [17], baixo custo [18], menor tensão residual e distorção [19], baixo teor de microinclusões [20], melhoria nas propriedades de ruptura em fluência [21] e capacidade de soldar grandes espessuras devido à maior penetração [22].

Em aços C-Mn, os cordões de solda geralmente possuem uma microestrutura formada por: ferrita primária (FP), ferrita com segunda fase (FS) e ferrita acicular (FA) em função das condições de resfriamento e composição química. Entretanto, quando a zona fundida é constituída predominantemente de ferrita acicular, tem-se uma das melhores condições microestruturais da solda, pois exige grande perda de energia para a propagação de uma trinca, 
na medida em que suas ripas de ferrita acicular obrigam a trinca a mudar de direção constantemente [8]. A avaliação da presença desses constituintes é de extrema importância para obtenção de uma correlação entre a microestrutura e as propriedades mecânicas.

Portanto, buscando entender melhor o processo A-TIG foram realizados testes desse tipo de soldagem comparando com TIG em um aço baixo carbono com microadição de molibdênio, com o objetivo de avaliar o incremento na profundidade dos cordões de solda, verificar as características morfológicas da microestrutura e os possíveis efeitos sobre as propriedades mecânicas observadas nas medidas de ultramicrodureza ao longo da espessura do cordão de solda na zona fundida.

\section{Material e métodos}

O material utilizado para os testes de soldagem foi um aço baixo carbono com microadição de molibdênio na condição de chapa laminada a quente com uma espessura de 3,2 mm. A composição química dessa liga é apresentada na Tabela 1.

A fonte de energia utilizada para as operações de soldagem foi uma fonte transistorizada multiprocessos (INVERSAL 300 da IMC). Nos testes de soldagem utilizou-se uma tocha refrigerada a água, o eletrodo era de tungstênio dopado com óxido de tório ( $\varnothing 3,2 \mathrm{~mm}$ ), a corrente de soldagem foi de 100 A e o comprimento do arco foi de $2 \mathrm{~mm}$. Esses parâmetros utilizados nos processos de soldagem foram baseados nos resultados obtidos por Liberato [23], onde o autor buscou caracterizar o efeito da soldagem A-TIG em um aço inoxidável ferrítico UNS S41003 com a variação dos parâmetros de soldagem, tipo e quantidade de fluxo. Foram realizadas soldagens TIG e A-TIG com fluxo de $\mathrm{Cr}_{2} \mathrm{O}_{3}$, sobre a chapa do aço em estudo sem chanfro, com o objetivo de verificar o comportamento da soldagem no metal base empregado, verificando a geometria do cordão de solda, a microestrutura e a ultramicrodureza. A preparação metalográfica dos corpos de prova consistiu no corte transversal do cordão de solda, onde realizou-se o lixamento, seguido de polimento mecânico com pasta de diamante. Para revelar a microestrutura do cordão de solda fez-se um ataque químico por imersão com Nital 4\%.

As análises da microestrutura foram conduzidas em um microscópio eletrônico de varredura (MEV), Modelo Quanta 250 FEG - fabricante FEI, com tensão de aceleração de $20 \mathrm{kV}$, spot size de 4,5 e distância de trabalho de $10 \mathrm{~mm}$.
As medidas de Ultramicrodureza foram realizadas em um Ultramicrodurômetro instrumentado, marca Shimadzu modelo DUH-211S, com indentador Berkovich. Realizou-se ciclos de carregamento/descarregamento com carga máxima de $100 \mathrm{gf}$, velocidade de 7,14 gf/s e $20 \mathrm{~s}$ de permanência em carga máxima, com todas as medidas conduzidas em pontos específicos da linha central da zona fundida ao longo da espessura na seção transversal do cordão de solda. Onde observou-se o comportamento das seguintes propriedades mecânicas:

- HT-115 - Dureza convencional em escala Berkovich que avalia a medida das diagonais da indentação, em função da carga máxima aplicada, após a completa remoção do indentador;

- Hit - Dureza da indentação que pode ser relacionada qualitativamente à tensão limite de escoamento plástico, ou seja, onde o esforço associado a penetração deixa de ser simplesmente elástico e tem-se o início da deformação plástica [24].

\section{Resultados e discussão}

Na Figura 1 é apresentado o aspecto microestrutural obtido via MEV do metal base, verifica-se uma microestrutura refinada com uma matriz predominantemente ferrítica (regiões de baixo relevo) associada a grãos com aspecto poligonal tendendo a acicular em conjunto com regiões perlíticas, identificadas pela morfologia lamelar, conforme destacado na Figura $1 b$.

As microestruturas encontradas na zona fundida (zona central) do cordão de solda das duas condições de soldagem (TIG e A-TIG) realizadas neste trabalho são apresentadas nas Figuras 2 e 3, respectivamente.

Na Figura 2 é apresentado o aspecto microestrutural resultante da soldagem TIG onde observa uma zona fundida com uma estrutura bem refinada e a predominância de ferrita acicular (FA), que são pequenos grãos não alinhados formados no interior dos grãos de austenita prévia [25,26], além de uma pequena fração de ferrita primária (FP), que são pequenos grãos com aspecto em blocos. Essa ferrita primária é uma ferrita poligonal que não está associada ao contorno de grão da austenita [25,26]. A presença dessa ferrita acicular favorece à tenacidade, pois evita caminhos preferenciais à propagação de trincas, exigindo grande perda de energia para a propagação de uma trinca, na medida em que suas ripas de ferrita acicular obrigam a trinca a mudar

Tabela 1. Composição química do aço em estudo (\% peso)

\begin{tabular}{ccccccccc}
\hline $\mathbf{C}$ & $\mathbf{M n}$ & $\mathbf{P}$ & $\mathbf{S}$ & $\mathbf{S i}$ & $\mathbf{C u}$ & $\mathbf{N i}$ & $\mathbf{C r}$ \\
\hline 0,082 & 1,606 & 0,016 & 0,008 & 0,005 & 0,014 & 0,009 & 0,020 \\
\hline $\mathbf{M o}$ & $\mathbf{S n}$ & $\mathbf{A l}$ & $\mathbf{N}$ & $\mathbf{T i}$ & $\mathbf{N b}$ & $\mathbf{V}$ & $\mathbf{F e}$ \\
\hline 0,155 & 0,003 & 0,028 & 0,006 & 0,002 & 0,014 & 0,005 & balanço \\
\hline
\end{tabular}



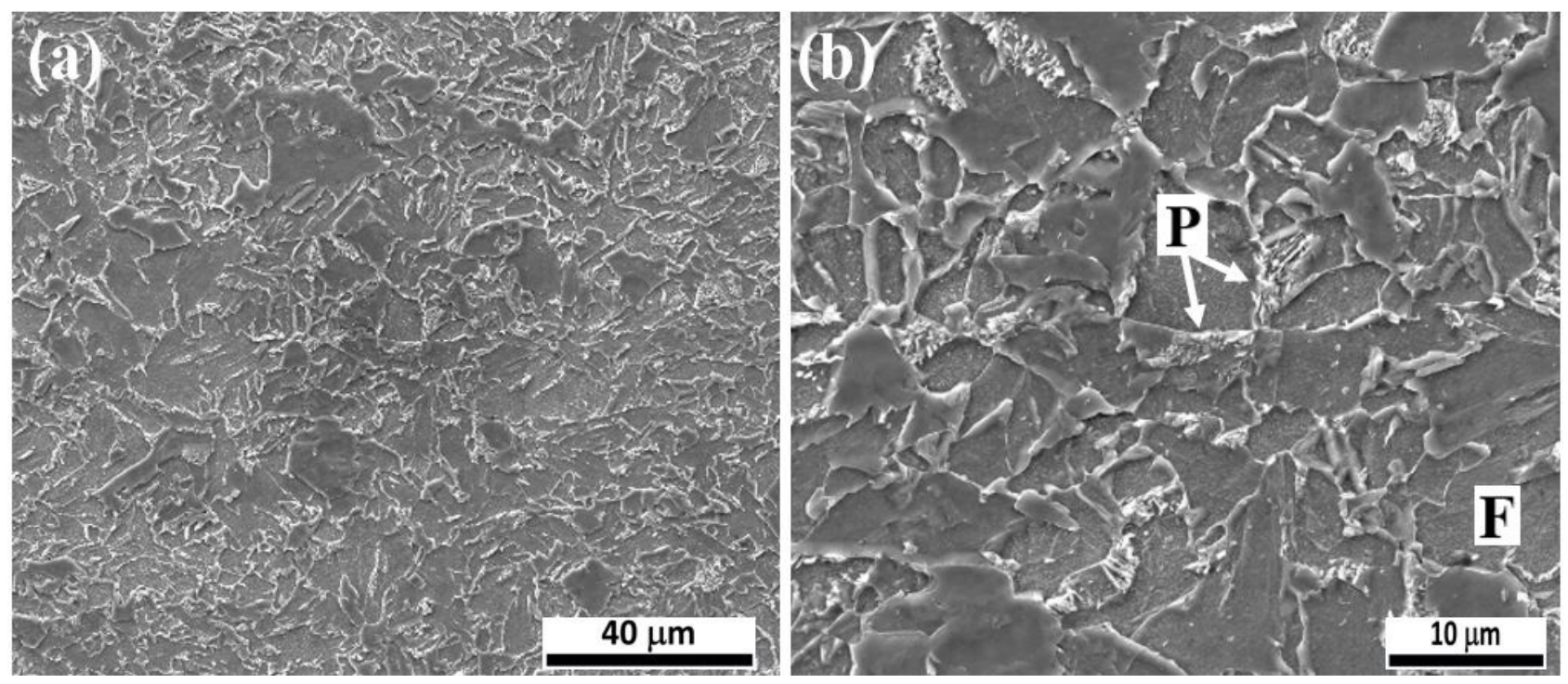

Figura 1. Microestrutura do metal base (a)1000x; (b)3000x; F = Ferrita; $P=$ Perlita
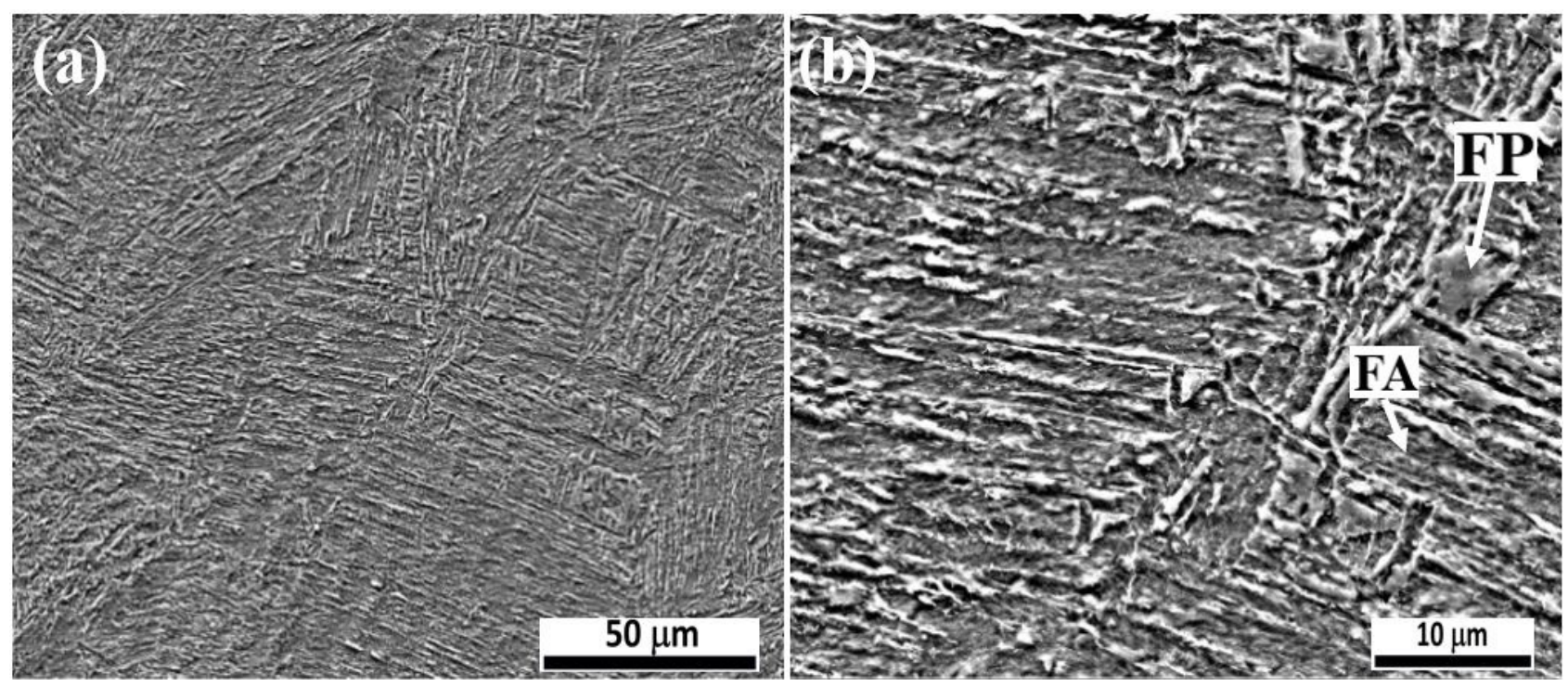

Figura 2. Microestrutura da zona fundida, TIG (a)800x; (b)3000x; FA = Ferrita acicular; FP = Ferrita primária.

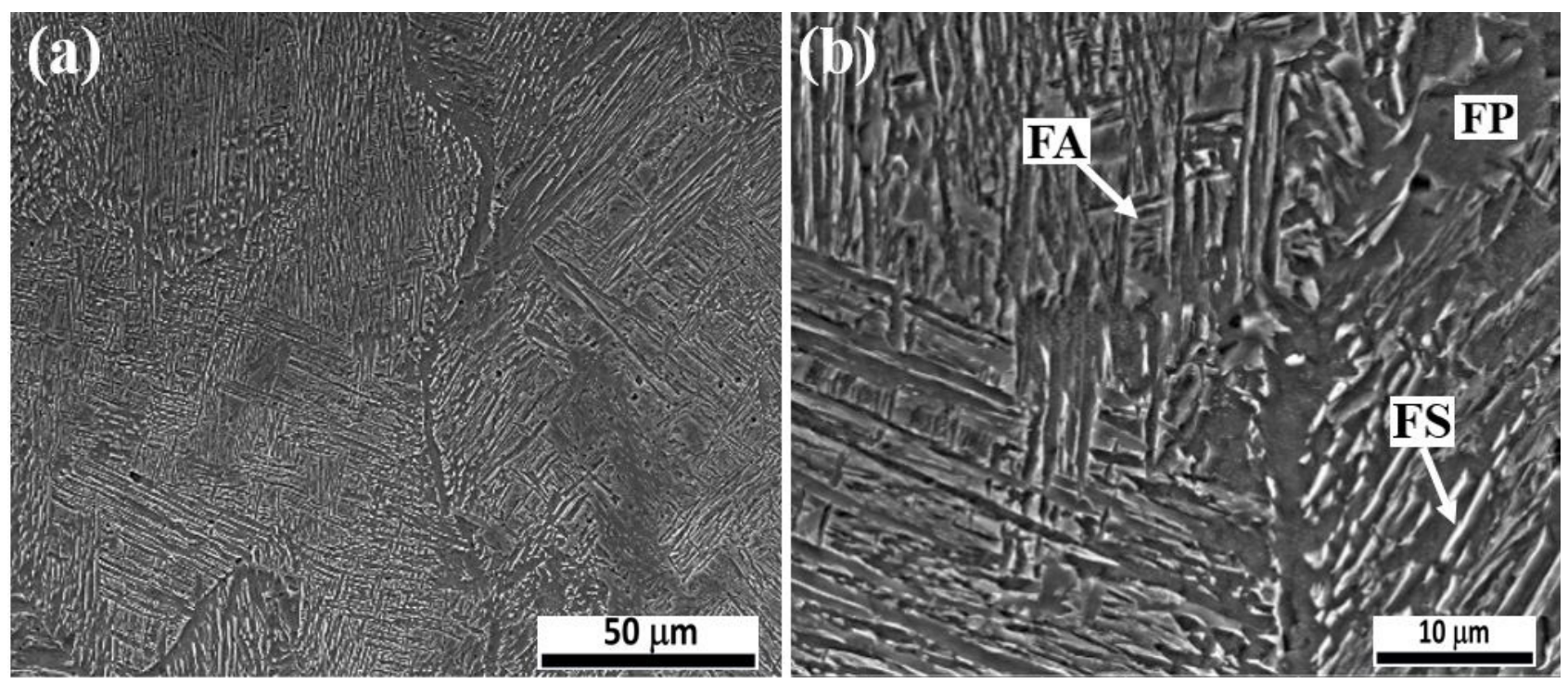

Figura 3. Microestrutura da zona fundida, A-TIG (a) 800x; (b) 3000x. FA= Ferrita acicular; FP=Ferrita primária; FS = Ferrita com segunda fase . 
de direção constantemente durante sua propagação [27]. A Figura 3 apresenta a microestrutura da zona fundida da soldagem A-TIG, onde observa-se a presença de ferrita acicular (FA), em conjunto da ferrita com segunda fase (FS) e da ferrita primária (FP). A ferrita de segunda fase alinhada possui uma morfologia de duas ou mais ripas paralelas de ferrita $[25,26]$, conforme destacado na Figura $3 b$.

Quando se compara as duas soldagens percebe-se que a TIG, por simplesmente comparação das micrografias, destaca-se uma aparente maior predominância de ferrita acicular, além de uma microestrutura mais refinada que naquela influenciada pelo processo com fluxo de $\mathrm{Cr}_{2} \mathrm{O}_{3}$. Esta possível predominância de ferrita acicular na zona fundida com vestígios de ferrita primária (FP) resultante da soldagem TIG, apesar de não quantificada, se reforça pelo fato da ferrita acicular presente na microestrutura da zona fundida da soldagem A-TIG coexistir junto a ferrita com segunda fase (FS) e ferrita primária (FP).

Com o intuito de verificar as variações na geometria do cordão de solda em ambas as amostras (Figura 4), foram calculadas as médias da largura e profundidade da zona fundida nos perfis dos cordões, onde obteve os valores apresentados na Tabela 2 juntamente com o respectivo desvio padrão. Esses resultados mostram que houve um aumento na profundidade e na largura do cordão de solda da amostra com adição do fluxo ativo de óxido de cromo $\left(\mathrm{Cr}_{2} \mathrm{O}_{3}\right)$. Não foram detectadas descontinuidades de soldagem do tipo mordeduras, porosidades e/ou trincas em ambas as amostras comparadas. Portanto, na soldagem realizada infere-se realmente um incremento da profundidade na solda A-TIG, como era esperado de acordo com a literatura [1-3]. O fluxo utilizado de $\mathrm{Cr}_{2} \mathrm{O}_{3}$ mesmo sendo simples permitiu que a zona termicamente afetada atingisse praticamente toda espessura da amostra como observado Figura 4b [9]. No mecanismo de convecção de Marangoni a razão da tensão superficial com o coeficiente de temperatura determina principalmente as forças do arco e a forma do cordão de solda. De acordo com o mecanismo, o fluxo dissolvido na poça de fusão pode alterar as propriedades da tensão superficial $(\gamma)$ durante a soldagem. Alguns trabalhos $[28,29]$ sugerem que os elementos ativos existentes na poça de fusão mudam $\delta \gamma / \delta \mathrm{T}$ de negativo

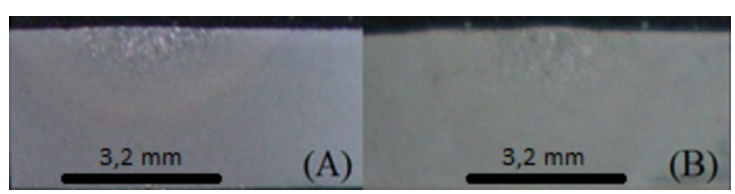

Figura 4. Análise macrográfica dos processos de soldagem (a) TIG; (b) A-TIG com fluxo de $\mathrm{Cr}_{2} \mathrm{O}_{3}$. para positivo quando a temperatura atinge um determinado valor crítico. A convecção de Marangoni impulsiona o fluxo de metal fundido radialmente, para o centro do cordão de solda, resultando em maior profundidade e menor largura do cordão de solda. No entanto, o coeficiente $\delta \gamma / \delta$ T negativo faz com que a convecção de Marangoni conduza o fluxo de metal radialmente para fora se a temperatura estiver abaixo deste valor crítico.

Este fenômeno faz com que o cordão de solda tenha alta profundidade no centro e aumento de largura na borda. Esse fato pode ser o responsável pelo incremento na largura do cordão da solda A-TIG em relação a TIG (Tabela 2). Os testes realizados no presente trabalho não permitem indicar claramente qual dos mecanismos (constrição do arco ou convecção de Marangoni) seria o mais relevante para as mudanças no perfil do cordão de solda associadas com a soldagem A-TIG $[5,10,11]$.

A análise de ultramicrodureza foi realizada ao longo da espessura, onde 0 é a superfície da solda, ou seja, o reforço, com o incremento da posição no sentido da raiz do cordão de solda. Pelos resultados obtidos, apresentados na Figura 5, observa-se que na face do cordão de solda, em ambos os processos utilizados, tem-se os maiores valores e que quanto mais próximo da raiz do cordão esses valores, se aproximam do valor do metal base. Esse comportamento também foi observado no trabalho de Pichumani e colaboradores que fizeram um estudo de correlação entre a microestrutura, microdureza e taxa de resfriamento em diferentes condições de soldagem, utilizando TIG e A-TIG com diversos fluxos [30]. A solda com fluxo (A-TIG) apresentou os maiores valores de dureza Berkovich (HT-115) ao longo da espessura (Figura 5a). Ocorre uma maior variação dos valores da dureza (HT-115) para a soldagem A-TIG, mais próximo da raiz do cordão de solda que para a TIG, quando comparada com o metal base. Esse fato pode ser um indicativo que a soldagem A-TIG atingiu maior profundidade do cordão de solda em função da maior densidade de energia resultante, que por consequência também influenciou no resultado de uma zona termicamente afetada maior, conforme observado nos valores superiores de Hit do metal base entre 2,25 e $3,0 \mathrm{~mm}$ ao longo da espessura. Como pode ser observado na Figura 4b, essa zona termicamente afetada seria um sombreado em completamente toda a espessura da amostra.

$\mathrm{Na}$ Figura $5 \mathrm{~b}$, tem os resultados encontrados para a propriedade denominada Hit, ou seja, dureza da indentação, onde verifica-se que essa propriedade teve maior influência quando compara os tipos de soldagem (TIG e A-TIG) com o metal base.

Tabela 2. Valores da média e desvio padrão da profundidade e largura dos cordões de solda

\begin{tabular}{cccccc}
\hline \multirow{2}{*}{ Amostra } & \multicolumn{2}{c}{ Largura (mm) } & & \multicolumn{2}{c}{ Profundidade (mm) } \\
\cline { 2 - 3 } \cline { 5 - 6 } & Média & Desvio Padrão & & Média & Desvio Padrão \\
TIG & 2,367 & 0,595 & 0,005 & 0,011 \\
A-TIG & 2,574 & 0,888 & 0,003 & 0,009 \\
\hline
\end{tabular}



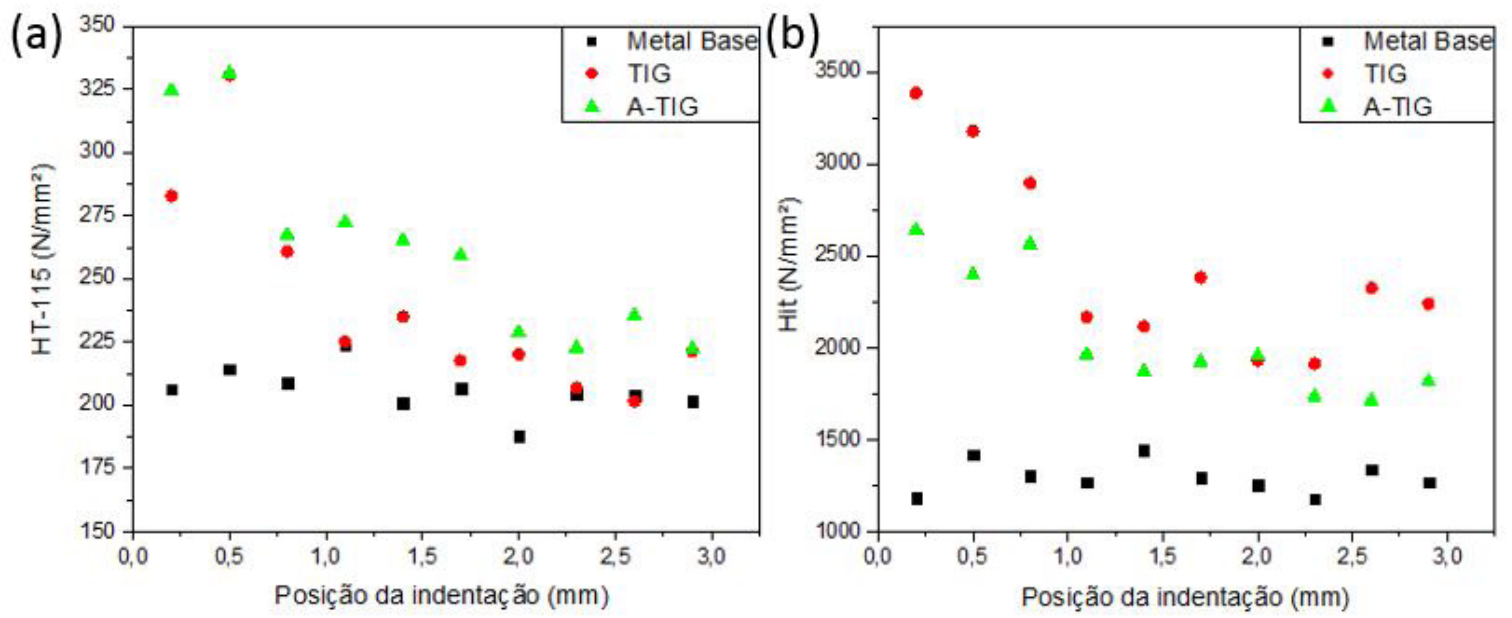

Figura 5. Propriedades mecânicas avaliadas no metal base e na zona fundida resultante da soldagem TIG e A-TIG: (a) Dureza Berkovich HT115; (b) Dureza da indentação - Hit (Posição da indentação: 0 = próximo ao reforço da solda; 3 = raiz da solda).

Entretanto o processo de soldagem TIG apresentou maiores valores para essa propriedade que o processo A-TIG, isso possivelmente está relacionado à microestrutura mais refinada, que promoveu maior resistência ao início da deformação plástica. As evidências macroestruturais e microestruturais do cordão de solda, quanto a penetração e refinamento microestrutural, em confronto com o perfil das propriedades mecânicas analisadas (HT-115 e Hit) para as duas soldagens executadas (TIG e A-TIG) trazem indicativos de que as taxas de resfriamento a que foram submetidas as zonas fundidas resultantes foram distintas. Visto que, a velocidade de resfriamento varia inversamente com a energia de soldagem, e mais significativamente em chapas de menor espessura [31]. Suportando que o motivo da microestrutura mais refinada na zona fundida da solda TIG seja relacionado ao ciclo térmico, pois esse ciclo depende fortemente, do material, geometria da junta e energia de soldagem. Como no presente trabalho a única variável alterada foi a energia de soldagem, que incrementou quando utilizou a soldagem A-TIG.

\section{Conclusão}

As microestruturas encontradas no cordão de solda foram: ferrita acicular (FA), ferrita com segunda fase (FS) e ferrita primária (FP), com aparente maior predominância para o microconstituinte FA encontrado principalmente na zona fundida da soldagem TIG.

$\mathrm{Na}$ soldagem A-TIG com fluxo de $\mathrm{Cr}_{2} \mathrm{O}_{3}$, obteve resultados significativos em termos de penetração comparando com a soldagem TIG, o que corrobora para comprovar a boa eficiência do processo de soldagem A-TIG. Entretanto, ocorre um pequeno incremento na largura do cordão dessa soldagem, que pode ter relação com a convecção de Marangoni.

Quanto às análises de ultramicrodureza, observa-se uma tendência coerente com a literatura, onde na face da solda obteve os maiores valores, em ambos processos utilizados, e esses resultados reduziram no sentido da raiz da solda para valores próximos aos encontrados no metal base. Esse comportamento tem relação com os efeitos térmicos promovidos pelos tipos de soldagem aplicados, na microestrutura.

\section{Agradecimentos}

Os autores agradecem a Companhia Siderúrgica Nacional (CSN) pela doação do aço, a UFMG pelos recursos para a realização dos processos de soldagem no LRSS/LabSEND, o IME pela utilização do MEV, a UFF pela disponibilização do ultramicrodurômetro instrumentado. A.S.P. agradece a bolsa de produtividade à pesquisa ( $\mathrm{PQ}-2)$ do $\mathrm{CNPq}$ (Processo 307798/2015-1). M.A.M.G agradece a bolsa de doutorado subsidiada pela CAPES vinculada ao PGCM/SE8-IME.

\section{Referências}

1 Vasantharaja P, Vasudevan M. Studies on A-TIG welding of Low Activation Ferritic/Martensitic (LAFM) steel. Journal of Nuclear Materials. 2012;421:117-123.

2 Singh AK, Dey V, Rai RN. Techniques to improveweld penetration in TIG welding (a review). Materials Today: Proceedings. 2017;4(2):1252-1259. http://dx.doi.org/10.1016/j.matpr.2017.01.145. 
3 Venkatesan JG, Sowmyasari M and Muthupandi V. Effect of ternary fluxes on depth of penetration in A-TIG welding of AISI 409 ferritic stainless steel. Procedia Materials Science. 2014;5:2402-2410.

4 Azevedo AGLD, Ferraresi VA, Faria SJP. Uma revisão sobre o processo de soldagem A-TIG. In: Faculdade de Engenharia Mecânica UFU. Simpósio de Pós-Graduação em Engenharia Mecânica. Uberlandia, Brasil. Uberlândia: UFU; 2006.

5 Lucas W, Howse DS. Activating flux-Increasing the performance and productivity of the TIG and plasma processes. Welding \& Metal Fabrication. 1996;64(1):11-17.

6 Paskell T, Lundin C, Castner H. GTAW flux increases weld joint penetration. Welding Journal. 1997;76(4):57-62.

7 Huang HY, Shyu SW, Tseng KH, Chou CP. Evaluation of TIG flux welding on the characteristics of stainless steel. Science and Technology of Welding and Joining. 2005;10(5):566-573.

8 Modenesi PJ, Apolinário ER, Pereira IM. TIG Welding with single-component fluxes. Journal of Materials Processing Technology. 2000;99:260-265.

9 Dong C, Zhu Y, Chai G, Zhang H. Preliminary study on the mechanism of arc welding with the activating flux. Japan: Joining and Welding Research Institute, Osaka University; 2004.

10 Berthier A, Paillard P, Carin M, Pellerin S, Valensi F. TIG and A-TIG welding experimental investigations and comparison with simulation part 2-arc constriction and arc temperature. Science and Technology of Welding and Joining. 2012;17(8):616-621.

11 Heiple CR, Roper JR. Mechanism for minor element effect on GTA fusion zone geometry. Welding Journal. 1982;61(4):97-120.

12 Novicki N, Buschinelli AJA, Paredes RSC. Amanteigamento por aspersão térmica na soldagem em operação de dutos de pequena espessura - estabilidade e penetração do arco voltaico. Soldagem \& Inspeção. 2011;16(3):243-255.

13 Lowke JJ, Tanaka M, Ushio M. Mechanisms giving increased weld depth due to a flux. Journal of Physics. D, Applied Physics. 2005;38:3438-3445.

14 Leconte S, Paillard P, Chapelle P, Henrion G, Saindrenan J. Effects of flux containing fluorides on TIG welding process. Science and Technology of Welding and Joining. 2007;12(2):120-126.

15 Anderson PCJ, Wiktorowicz R. Improving productivity with A-TIG welding. USA: TWI and Air Products; 1996.

16 Abinaya S, Verma DK, Noorullah D, Sundararaj P. A-TIG Process and Mechanism-Review Paper IJSRD International Journal for Scientific Research \& Development. 2019;7(1):2321-0613

17 Vasudevan M, Bhaduri AK, Raj B, Prasad Rao K. Genetic algorithm based computational model for optimizing the process parameters in A-TIG welding of 304LN and 316LN stainless steels. Materials and Manufacturing Processes. 2007;22(5):641-649.

18 Chandrasekhar N, Vasudevan M. Intelligent modeling for optimization of A-TIG welding process. Materials and Manufacturing Processes. 2010;25(11):1341-1350.

19 Maduraimuthu V, Vasudevan M, Muthupandi V, Bhaduri AK, Jayakumar T. Study of the effect of activated flux on the microstructure and mechanical properties of mod. 9Cr-1Mo steel. Metallurgical and Materials Transactions. B, Process Metallurgy and Materials Processing Science. 2012;43(1):123-132.

20 Lin H-L, Wu T-M. Effects of activating flux on weld bead geometry of Inconel 718 alloy TIG welds. Materials and Manufacturing Processes. 2012;27:1457-1461.

21 Sakthivel T, Vasudevan M, Laha K, Parameswaran P, Chandravathi KS, Paneerselvi S, et al. Creep-rupture behavior of 9Cr-1.8W-0.5Mo-VNb P92 ferritic steel weld joint. Materials Science and Engineering A. 2014;591:111-120.

22 Vidyarthy RS, Dwivedi DK. Activating flux tungsten inert gas welding for enhanced weld penetration. Journal of Manufacturing Processes. 2016;22:211-228. http://dx.doi.org/10.1016/j.jmapro.2016.03.012.

23 Liberato FM. Soldagem A-TIG em Aços Inoxidáveis Ferríticos UNS S41003 [dissertação]. Belo Horizonte: UFMG; 2013.

24 Shimadzu. DUH-211/DUH-211S instruction manual: dynamic ultra-micro hardness tester. Kyoto: Shimadzu Corporation; 2009.

25 Dallan CB, Damkroger BK. Characterization of welds. In: Davis JR. ASM handbook: welding, brazing, and soldering. Metals Park: American Society for Metals; 1993. p.202-209.

26 Colpaert H. Metalografia dos produtos siderúrgicos comuns. $4^{\text {a }}$ ed. São Paulo: Edgard Blucher, 2008. 
Gurgel et al.

27 Zhang T, Li Z, Young F, Kim HJ, Li H, Jing H, et al. Global progress on welding consumables for HSLA steel. ISIJ International. 2014;54(7):1472-1484.

$28 \mathrm{Lu} \mathrm{S}$, Fujii H, Nogi K. Marangoni convection and weld shape variations in $\mathrm{Ar}-\mathrm{O}_{2}$ and $\mathrm{Ar}-\mathrm{CO}_{2}$ shielded GTA welding. Materials Science and Engineering. 2004;380:290-297.

29 Fujii H, Lu S, Sato T, Nogi K. Effect of Oxygen Content in $\mathrm{He}_{-} \mathrm{O}_{2}$ Shielding Gas on Weld Shape in Ultra Deep Penetration TIG. Transactions of JWRI. 2008;37(1):19-26.

30 Pichumani S, Srinivasan R. Effect of Pulsed Current and Activated Flux with TIG Welding on the Microstructure and Mechanical Properties of Al-SiC Composite. Indian Journal of Science and Technology. 2015;8(27):1-6.

31 Modenesi PJ, Marquer PV, Santos DB. Introdução à Metalurgia da Soldagem. Belo Horizonte: Universidade Federal de Minas Gerais; 2006.

Recebido em: 26 Jun. 2019

Aceito em: 18 Maio 2021 